\title{
Origami-Inspired Smart Building Skin ${ }^{\dagger}$
}

\author{
Silvia Andreozzi 1, Gaia Ilenia Bessone 2, Matteo Botto Poala 3, Martina Bovo 4, \\ Silvia Fernandez De Alaiza Amado 4, Emanuele Giargia 5, Alessandro Niccolai 6 , \\ Viola Papetti ${ }^{6}$, Francesco Braghin ${ }^{7}$ and Stefano Mariani ${ }^{8, *}$
}

1 Politecnico di Torino, Mathematical Engineering, Corso Duca degli Abruzzi, 24, 10129 Torino, Italy; silvia.andreozzi@hotmail.it

2 Politecnico di Milano, Interior Design, Architecture, Piazza L. da Vinci 32, 20133 Milan, Italy; gaia2992@yahoo.it

3 Politecnico di Torino, Architecture, Corso Duca degli Abruzzi, 24, 10129 Torino, Italy; matteo.botto@gmail.com

4 Politecnico di Milano, Architecture, Piazza L. da Vinci 32, 20133 Milan, Italy; bovomartina@gmail.com(M. B.), silvia.fernandezdealaiza@mail.polimi.it(S.A.)

5 Politecnico di Torino, Mechanical Engineering, Corso Duca degli Abruzzi, 24, 10129 Torino, Italy; emag992@gmail.com

6 Politecnico di Milano, Mechanical Engineering, Department of Mechanical Engineering, Piazza L. da Vinci 32, 20133 Milan, Italy; ale.nicc@gmail.com, viola.papetti46@gmail.com

7 Politecnico di Milano, Department of Mechanical Engineering, Piazza L. da Vinci 32, 20133 Milan, Italy; francesco.braghin@polimi.it

8 Politecnico di Milano, Department of Civil and Environmental Engineering, Piazza L. da Vinci 32, 20133 Milan, Italy

* Correspondence: stefano.mariani@polimi.it; Tel.: +39-02-23994279

+ Presented at the 3rd International Electronic Conference on Sensors and Applications, 15-30 November 2016; Available online: https://sciforum.net/conference/ecsa-3.

Published: 28 November 2016

\begin{abstract}
We propose the design of an environment-responsive, deployable origami-inspired structure to be used as a smart building skin. The folding structure is composed by rigid panels connected to each other through hinge-like connectors. The overall degree of openness of the whole structure is adjusted in response to variations of environmental parameters like lighting and temperature, recorded by a network of embedded sensors. The geometry and kinematics of the origami are selected so as the deployment of each module can be induced at some key points that only slide along a linear axis; in this way, electric motors with a positional control logic can prove efficient. By properly tuning the properties of each panel mounted on the frames, the proposed solution can be adopted as a shading or light refraction system, thus improving the comfort of the building interiors. Through digital prototyping and small-scale models, the effectiveness of the proposed solution is assessed. Some site-specific applications are finally discussed from the self-sensing, self-actuation and self-powering viewpoints.
\end{abstract}

Keywords: adaptive systems; deployable structures; modular origami structures; self sensing and actuation

(C) 2016 by the authors. Licensee MDPI, Basel, Switzerland. This article is an open access article distributed under the terms and conditions of the Creative Commons Attribution (CC BY) license (http://creativecommons.org/licenses/by/4.0/). 